\title{
Forkheads, winged helices, and gastrointestinal epithelium
}

Kaestner KH, Silberg DG, Traber PG, Schütz G. The mesenchymal winged helix transcription factor Fkh6 is required for the control of gastrointestinal proliferation and differentiation. Genes Dev 1997;11:1583-95.

\section{Abstract}

The winged helix transcription factor Fkh6 is expressed in the mesoderm of the gastrointestinal tract directly adjacent to the endoderm-derived epithelium. Homozygous null mice for Fkh6 showed postnatal growth retardation secondary to severe structural abnormalities of the stomach, duodenum, and jejunum. Dysregulation of epithelial cell proliferation in these organs resulted in an approximately fourfold increase in the number of dividing intestinal epithelial cells and marked expansion of the proliferative zone. As a consequence, the tissue architecture of the stomach and small intestine was distorted, with abnormal crypt structure, formation of mucin filled cysts, and lengthening of villi. Changes in the cellular phenotype and composition of the gastric and intestinal epithelia also suggests that epithelial cell-lineage allocation or differentiation may be affected by loss of Fkh6. From the analysis of a number of potential signaling molecules, we found $B m p 2$ and $B m p 4$ expression reduced in the gastrointestinal tract of Fkh6 mutant mice, suggesting that Fkh6 directs a signaling cascade that mediates communication between the mesenchyme and endoderm of the gut to regulate cell proliferation.

\section{Comment}

Developmental abnormalities of the gastrointestinal tract are an important cause of morbidity in infants. Oesophageal atresia (incidence 1 in 2000 live births), Hirschsprung's disease ( 1 in 5000), and duodenal atresia form much of the case work of a paediatric surgeon, yet virtually nothing is known about the molecular mechanisms that control gastrointestinal morphology. The article by Kaestner et al is the first to show how a regulatory gene in the embryo can alter gastrointestinal structure. They report morphological changes in the development of villi in the upper small intestine and the stomach epithelia.

Mesenchymal cells adjacent to the endoderm are central to the normal ontogeny of the gastrointestinal epithelium. ${ }^{12}$ Kaestner et al examined a regulatory protein, Fkh6 (Forkhead homolog 6), which is expressed only in the mesenchyme adjacent to the developing endoderm that is destined to become epithelium. Fkh6 was therefore a candidate gene in the regulation of gastrointestinal development. ${ }^{3}$ Fkh6 is a member of the winged helix family of transcription factors, so called because of their appearance when bound to the DNA whose transcription they regulate. ${ }^{4}$
Fkh6 was deleted from the genome of embryonic stem cells by targeting the gene with a neomycin resistance gene, the standard technique for producing knockout mice through homologous recombination. Homozygous null mice $\left(\mathrm{Fkh}^{-/-}\right)$did not express $F k h 6$ mRNA. Importantly, they also displayed changes in gastrointestinal epithelial cell proliferation and differentiation. Normally in fetal mice, the small intestine develops from a stratified into a columnar epithelium. This transition is associated with the invagination and condensation of mesenchyme. Rudimentary villi then grow, the timing of which was delayed in the $\mathrm{Fkh}^{-1-}$ mice. When villi did appear, they were less well developed and were shorter, wider and fewer in number. Although all four epithelial cell types (enterocytes, goblet cells, enteroendocrine cells, and Paneth cells) were present in the null mice, goblet cell numbers were increased in the proximal intestine. Normally there are fewer goblet cells in the proximal than in the distal intestine. In $\mathrm{Fkh}^{-/-}$mice, the changes in the villous pattern resulted in poor weight gain after birth, presumably because of poor nutrient absorption. Villous development did not improve after birth. By postnatal day 12, crypts appeared in both the wild type and null mice; however, in the Fkh $6^{-/-}$mice, the crypt compartment was enlarged and the crypts were often branched. These are the classic features of increased epithelial cell proliferation. When null mice reached an age of 50 days or more, the increased proliferation had resulted in longer villi. The pattern in the stomach also revealed changes characteristic of poorly controlled epithelial cell proliferation. The glandular epithelium was expanded and, instead of glands being orderly, they were branched within the gastric mucosa. Surface cells were also vacuolated.

Direct staining for epithelial cell proliferation showed that dividing cells were not confined to focal areas either in the stomach or the small intestine, where they normally localise to the intervillous regions. These areas eventually become the base of crypts. In the $\mathrm{Fkh}^{-/-}$mice, proliferating cells were scattered throughout the villous surface. It seems likely therefore that $F k h 6$ induces the expression of factor(s) in the mesenchyme cells that regulate epithelial cell division. Despite an exhaustive search for the intermediate factor that signals between the mesenchyme and the epithelium, ${ }^{5}$ its identity is not yet known. However, it is likely that Kaestner et al will discover it in the near future. They have examined homologues of the extracellular proteins decapentaplegic and wingless that control endoderm expression in the midgut of Drosophila.$^{67}$ The expression of two such homologues, $B m p 2$ and $B m p 4,{ }^{7}$ was reduced in the $\mathrm{Fkh}^{-/-}$mice. Experiments using mesenchymal cells derived from $\mathrm{Fkh}^{-/-}$mice cultured in vitro may provide further clues.

Another aspect of the work that remains unexplained is the proximal localisation of the changes, which were present only in the stomach and small intestine. The distal small intestine and colon appeared normal. Yet, the Fkh6 gene is expressed throughout the gastrointestinal mesenchyme. How proliferation can be regulated normally in the distal but not the proximal gastrointestinal tract in $\mathrm{Fkh}^{-/-}$ 
mice is puzzling. This may be related to as yet undiscovered regulatory proteins in the mesenchymal cells. Such proteins may be distributed unevenly along the gut.

Although Kaestner et al's article leaves many questions unanswered, it is a step in our understanding of the molecular development of the mammalian gastrointestinal tract. We can expect that over the next decade this field will increase in complexity. Eventually we will know the molecular defects responsible for the gut malformations that are such a central part of neonatal practice.

I R SANDERSON

Developmental Gastrointestinal Laboratory,

Combined Program in Pediatric Gastroenterology and Nutrition,

Massachusetts General Hospital, Boston, USA
1 Louvard D, Kedinger M, Hauri H-P. The differentiating intestinal epithelial cell: establishment and maintenance of functions through interactions between cellular structures. Annu Rev Cell Biol 1992;8:157-95.

2 Kedinger M, Simon-Assmann PM, Lacroix B, Marxer A, Hauri H-P, Haffen $\mathrm{K}$. Fetal gut mesenchyme induces differentiation of cultured intestinal endodermal and crypt cells. Dev Biol 1986;113:474-83.

3 Kaestner KH, Bleckmann SC, Monaghan AP, Schlöndorff J, Mincheva A, Lichter P, et al. Clustered arrangement of winged helix genes fkh-6 and MFH-1: possible implications for mesoderm development. Development 1996;122:1751-8.

4 Clark KL, Halay ED, Lai E, Burley SK. Co-crystal structure of the HNF-3/ forkhead DNA-recognition motif resembles histone H5. Nature 1993;364: 412-20.

5 Podolsky DK. Peptide growth factors in the gastrointestinal tract. In: Johnson LR, ed. Physiology and the gastrointestinal tract. New York: Raven Press, son LR, ed. Phys

6 Bienz M. Homeotic genes and positional signalling in the Drosophila viscera. Trends Genet 1994;10:22-6.

7 Bitgood MJ, McMahon AP. Hedgehog and Bmp genes are coexpressed at many diverse sites of cell interaction in the mouse embryo. Dev Biol 1995; 172:126-8. 